\title{
Response to:
}

- Di Puccio F, Giacomarro D, Mattei L, Pittiruti M, Scoppettuolo G: Experimental study on the chemico-physical interaction between a two-component cyanoacrylate glue and the material of PICCs. The Journal of Vascular Access 2017:0-0.

Full title: Tissue adhesive and chlorhexidine gluconate interaction: implications for vascular access device securement

Short title: $\mathrm{CHG}$ and glue

Authors: Amanda J Ullman 1,2,3,4, Tim R Dargaville 1,5, Claire M Rickard 1,2,3

${ }^{1}$ Alliance for Vascular Access Teaching and Research Group, Menzies Health Institute Queensland, Griffith University, Australia

${ }^{2}$ School of Nursing and Midwifery, Griffith University, Australia

${ }^{3}$ Centre for Clinical Nursing, Royal Brisbane and Women's Hospital, Australia

${ }^{4}$ Paediatric Critical Care Research Group, Lady Cilento Children's Hospital, Australia Institute of Health and Biomedical Innovation, Queensland University of

Technology, Australia

\section{Corresponding author:}

Dr Amanda Ullman

N48, Menzies Health Institute Queensland

Griffith University

Nathan, Queensland, Australia 4111

Phone: +61 737356462

Email: a.ullman@griffith.edu.au

Word count: 464

Keywords: tissue adhesive; securement; dressing; skin; wounds 
The growing evidence supporting tissue adhesive (TA), or medical-grade superglue, to promote vascular access security has resulted in rapid uptake in clinical settings $[1,2]$. TA manufacturers are exploring different chemical compositions to optimise viscosity and strength while maintaining biocompatibility. However, TAs are being applied within already complex clinical situations. Many other chemicals are already being used, and interactions between current practices and emerging technologies need to be examined. We read with interest Di Puccio and colleagues'[1] laboratory study demonstrating the safety of applying TA onto a range of uncoated polyurethane peripherally inserted central catheters (PICCs), without adversely affecting the catheter surfaces or mechanical strength. This safety data are reassuring for clinicians currently applying TA onto PICCs made of these materials, however the interaction with silicone demonstrates further testing with anti-microbial and anti-thrombogenic materials is important.

Further guidance regarding the application of TA is necessary, to ensure its success in the heterogeneous clinical settings where TA is being applied. Chlorhexidine gluconate $(\mathrm{CHG})$ is a vital antiseptic used in combination with alcohol for skin decontamination [3]. What is currently unknown from the literature and clinical practice is whether there is potential for adverse interaction between TA and CHG at vascular access insertion sites.

From a chemical perspective, CHG may interact with TAs in two ways. Firstly, the mechanism in which cyanoacrylate TA polymerizes is based on trace amounts of water on skin or in the air initiating the chemical reaction. Both the chlorhexidine and 
gluconate components of CHG have the potential to act similarly to water and commence the cyanoacrylate TA reaction process. Thus, in the presence of CHG TA's hardening reaction may be accelerated which could, theoretically, change the adhesive strength. The second potential interaction is with the alcohol component of CHG (usually isopropanol), which if not fully allowed to evaporate before application of TA may possibly also affect the chemical reaction. Preliminary experiments on healthy volunteers showed 2-octyl cyanoacrylate (Dermabond ${ }^{\mathrm{TM}}$, Ethicon, US) reacted faster and with greater heterogeneity on skin freshly swabbed with $2 \%$ CHG with $70 \%$ isopropyl alcohol (SoluPrep ${ }^{\mathrm{TM}}$ Swab; 3M, US), compared with nonswabbed skin. The same TA applied to swabbed then dried skin reacted faster than the non-swabbed control, but only at the skin surface.

Dermatitis injuries caused by inadequate CHG drying time prior to dressing application are common[4]. Vascular access device insertion and dressing change procedures can be harried, due to non-compliant patients (e.g., paediatrics) and staffing shortages. If TA becomes a primary securement method for vascular access devices, clinicians must ensure adequate CHG drying time, prior to TA application. Otherwise, this new innovation, instead of promoting device security, may possibly be associated with accidental dislodgement and consequential treatment disruption.

Financial support: None to declare

Conflicts of interest: Griffith University has received investigator-initiated research grants from tissue adhesive and dressing manufacturers (3M, Adhezion, BD, Centurion Medical Products) to support the research of AJU, unrelated to the current 
manuscript. Griffith University has received investigator-initiated research/education grants on CMR's behalf from tissue adhesive and dressing manufacturers (3M, Adhezion, BBraun, BD-Bard; Centurion Medical Products; Entrotech; Medtronic; Teleflex), and consultancy payments from tissue adhesive and dressing manufacturers (3M, BBraun, BD-Bard, ResQDevices).

\section{References:}

1. Di Puccio, F., et al., Experimental study on the chemico-physical interaction between a two-component cyanoacrylate glue and the material of PICCs. The Journal of Vascular Access, 2017: p. 0-0.

2. Chan, R.J., et al., Central venous Access device SeCurement And Dressing Effectiveness for peripherally inserted central catheters in adult acute hospital patients (CASCADE): a pilot randomised controlled trial. Trials, 2017. 18(1): $\mathrm{p}$. 458.

3. Mimoz, O., et al., Skin antisepsis with chlorhexidine-alcohol versus povidone iodine-alcohol, with and without skin scrubbing, for prevention of intravascular-catheter-related infection (CLEAN): an open-label, multicentre, randomised, controlled, two-by-two factorial trial. Lancet, 2015.

4. Broadhurst, D., N. Moureau, and A.J. Ullman, Central venous access devices site care practices: an international survey of 34 countries. J Vasc Access, 2016. 17(1): p. 78-86. 